Delft University of Technology

\title{
Land CSEM impulse responses in simple layered models
}

Slob, E.; Ziolkowski, A.

DOI

10.3997/2214-4609.201802585

Publication date

2018

Document Version

Final published version

Published in

24th European Meeting of Environmental and Engineering Geophysics

\section{Citation (APA)}

Slob, E., \& Ziolkowski, A. (2018). Land CSEM impulse responses in simple layered models. In M. J. Senos Matias, \& F. Santos (Eds.), 24th European Meeting of Environmental and Engineering Geophysics EAGE. https://doi.org/10.3997/2214-4609.201802585

\section{Important note}

To cite this publication, please use the final published version (if applicable).

Please check the document version above.

\section{Copyright}

Other than for strictly personal use, it is not permitted to download, forward or distribute the text or part of it, without the consent of the author(s) and/or copyright holder(s), unless the work is under an open content license such as Creative Commons.

\section{Takedown policy}

Please contact us and provide details if you believe this document breaches copyrights.

We will remove access to the work immediately and investigate your claim. 
Green Open Access added to TU Delft Institutional Repository 'You share, we take care!' - Taverne project

\section{https://www.openaccess.nl/en/you-share-we-take-care}

Otherwise as indicated in the copyright section: the publisher is the copyright holder of this work and the author uses the Dutch legislation to make this work public. 


\section{Tu 24B 07}

\section{Land CSEM Impulse Responses in Simple Layered Models}

E. Slob ${ }^{1 *}$, A. Ziolkowski ${ }^{2}$

${ }^{1}$ Delft University of Technology, ${ }^{2}$ University of Edinburgh

\section{Summary}

We argue that in a half space model with a single layer, the electric field impulse response at different offsets can be understood physically. Diffusive fields travel along paths like wavefields but are not localised in time. Each event that is measured comes from a path with the least attenuation, which corresponds to early arrival. The most prominent TE mode contribution to the detection of a subsurface layer comes from the coupling of the airwave with the subsurface. The most prominent contribution from the TM mode comes from the path through the resistive layer with almost vertical diffusion paths between the surface and the target. The arrival time of the peak of the event from a conductive layer can be understood from this reasoning. The arrival time of the peak of the event from a resistive layer can be understood from this reasoning when the offset is not too large compared with burial depth of the target layer. These predictions lead to estimates of depth to target layer with an error of less than $5 \%$ and the resistivity of the resistive layer of less than $50 \%$. The information contained in the impulse responses suggests that inversion would yield good results. 


\section{Introduction}

Controlled Source Electromagnetic (CSEM) as well as magneto-telluric (MT) measurements have a long history in several commercial applications with mixed results (den Boer et al., 2000; Sheard et al., 2005; Ziolkowski et al., 2007). With the advent of the CSEM method for hydrocarbon exploration in the marine environment, high power sources and high-quality sensors and receivers have become available. The source strength has increased by a factor of ten and the noise floor of the receivers has been reduced by the same factor compared with the situation of seven years ago (Hanssen et al., 2017). Developments of land CSEM equipment lag behind but are continuing as well. In combination with the increased computing power, the controlled source electromagnetic method is now capable of delivering detailed resistivity structures in joint inversion with seismic data (Panzner et al., 2016).

For CSEM, source and receiver control are essential for obtaining true amplitude measurements of electric fields that can be converted to the Earth's impulse responses. This is important because such measurements can be understood physically from diffusion paths through the subsurface of which the ones that have the lowest attenuation can be measured. We use physical arguments to predict the behaviour of the TE mode and TM mode parts of the electric field impulse responses in models with a target layer in a half space. The target layer can be resistive, e.g., in the case of $\mathrm{CO}_{2}$ storage, or conductive, e.g. in the case of a hot brine filled aquifer for geothermal use. We show numerical electric field impulse responses for parallel broadside receivers, which have an offset perpendicular to the field and source direction, and for inline receivers, which have an offset in the line along the vector direction of the source and the receiver. We evaluate to which extent the physical explanation of the numerical results based on geometrical considerations explain the features in the data for the two modes that together make up the electric field at the surface.

\section{The transient controlled-source electromagnetic method on land}

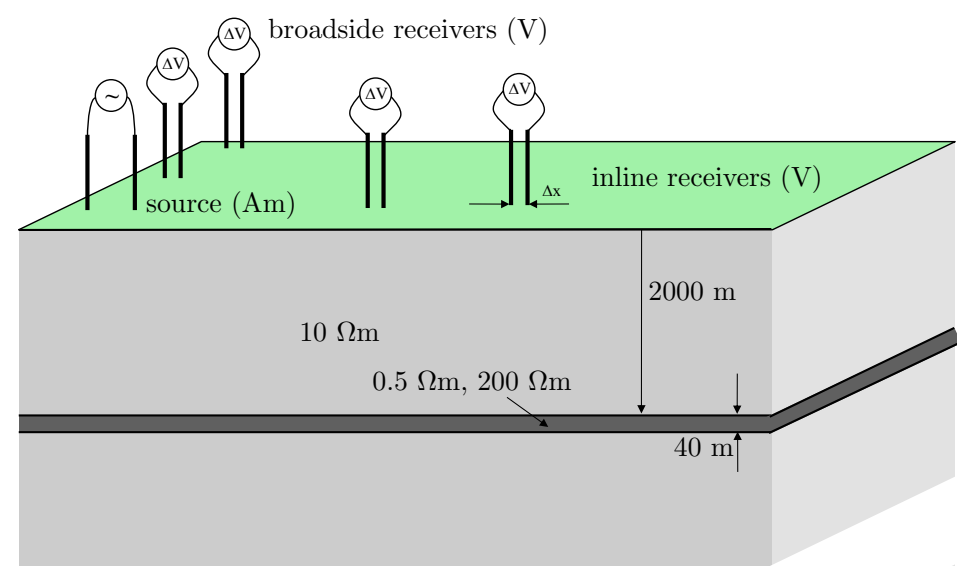

Figure 1 CSEM configuration with the source, parallel broadside and inline receivers on the surface.

Figure 1 shows two typical land acquisition line-configurations where vector components of the electric current source and of the electric field receivers are in the same direction. These are where the offset is perpendicular to or along the vector direction of source and receivers and they are called the broadside and inline configurations, respectively. Figure 1 indicates a simple model with a half space and a layer at $2 \mathrm{~km}$ below that surface that is either more resistive or more conductive than the half space. A more resistive layer could be a hydrocarbon bearing layer or a layer used for $\mathrm{CO}_{2}$ storage, whereas a more conductive layer could be a hot brine filled aquifer of possible interest for heating or the generation of geothermal energy.

Modelling codes to generate data for such configurations are available (Hunziker et al., 2015; Werthmüller, 2017). Those works give a deconstruction of the electromagnetic field in terms of TE and TM modes. Physically, it means that the TE mode field is associated with eddy currents that lie entirely in the horizontal plane, while the TM mode is associated with eddy currents that lie in any plane that con- 
tains the vertical axis. From the theoretical study of Slob et al. (2010) we know that under the diffusive approximation the airwave is entirely TE mode. Any field that is connected to the vertical component of the electric field and diffuses along a horizontal direction is entirely TM mode. With these two notions we can understand the impulse responses of inline and broadside electric field data from the model depicted in Figure 1. Because it is useful to evaluate separately the contributions from the TE and TM mode parts in the total electric field we have computed the separately. The mode separation comes with an apparent singularity. In each mode it is the sae but with opposite sign. When the total field is computed the result is zero, but when each mode is computed separately, it must be removed to see the true contribution from each mode. This is done by subtracting the result at zero radial wavenumber from each mode. Because diffusive fields can be understood as a sum of scaled wavefields through the correspondence principle (de Hoop, 1996; Mittet, 2015) we give three descriptions for important paths through the model of Figure 1 that will lead to significant contributions in the data.

In a half space, the field through the air and the ground along the surface are the only signals measured at the surface. The field through the air is the airwave and travels at speed of light and its amplitude drops by offset to the power three. The field through the ground is the direct field whose peak amplitude arrives at $t_{\text {peak }}=\mu_{0} r^{2} /(10 \rho)$, where $\rho$ is the half space resistivity, $\mu_{0}$ is the free space magnetic permeability and $r$ is offset. Its peak amplitude drops by offset to the power five (Ziolkowski et al., 2007). The inline direct field is entirely TM mode and the broadside field is entirely TE mode.

The airwave couples the TE mode to subsurface diffusion, which a travel path exists where the TE mode part of the field diffuses down from the source to the layer after which it reflects and diffuses up to the surface where it is converted into an airwave that travels horizontally along the surface at the speed of light. The reciprocal path also exists. The airwave arrives at all receivers without delay relative to sampling time. If this is a reasonably good approximation we expect that the amplitude of this part of the field only depends on offset and that the arrival time of the signal peak depends only on the two-way vertical diffusion time and shows no delay as a function of offset.

The TM mode does not have a significant airwave but the TM mode part of the field that diffuses down almost vertically in the plane spanned by the source vector direction and the vertical axis generates a strong vertical component electric field inside a resistive layer that diffuses through the resistive layer with high speed and correspondingly low attenuation, it diffuses up almost vertically and is recorded at the surface. The inline measurement configuration provides such a path, whereas the broadside configuration does not. When the direct wave travels more than two times the depth to resistive layer the TM mode path through the resistive layer can become stronger than the direct field in the inline measurements. At which offset this happens depends on the resistivity contrast between half space and resistive layer. For the broadside measurement we cannot make a simple prediction.

\section{Numerical examples}

First we look at the results of the half space with the conductive layer at $5 \mathrm{~km}$ and $10 \mathrm{~km}$ offsets. The electric field impulse responses for the conductive layer are shown in Figure 2. The top row shows results at $5 \mathrm{~km}$ offset and the bottom row at $10 \mathrm{~km}$ offset; the left column shows inline field and the right column shows broadside fields. The half space response is shown in black solid lies and the layered half space response in green solid lines. The contributions from the TE and TM modes are shown in blue and red dashed lines, respectively. The first observation is that the TE mode has opposite sign in inline configuration to the broadside configuration and that the TM mode is virtually absent in broadside.the moment of the peak amplitude in the TE mode is at $220 \mathrm{~ms}$ in all four plots whereas we would predict the peak to occur at $200 \mathrm{~ms}$. This would lead to an estimated depth to conductive layer of $2090 \mathrm{~m}$, which is an error of less than 5\%. It is clear that the conductive layer is better visible in the broadside data than in the inline data. Inline the conductive layer becomes strongly visible at very large offsets when the TM mode diffusion times are long as can be seen in the bottom left plot.

The electric field impulse responses for the resistive layer are shown in Figure 3 with the same line and colour scheme as used in Figure 2. Now we see that the inline response is almost entirely determined 

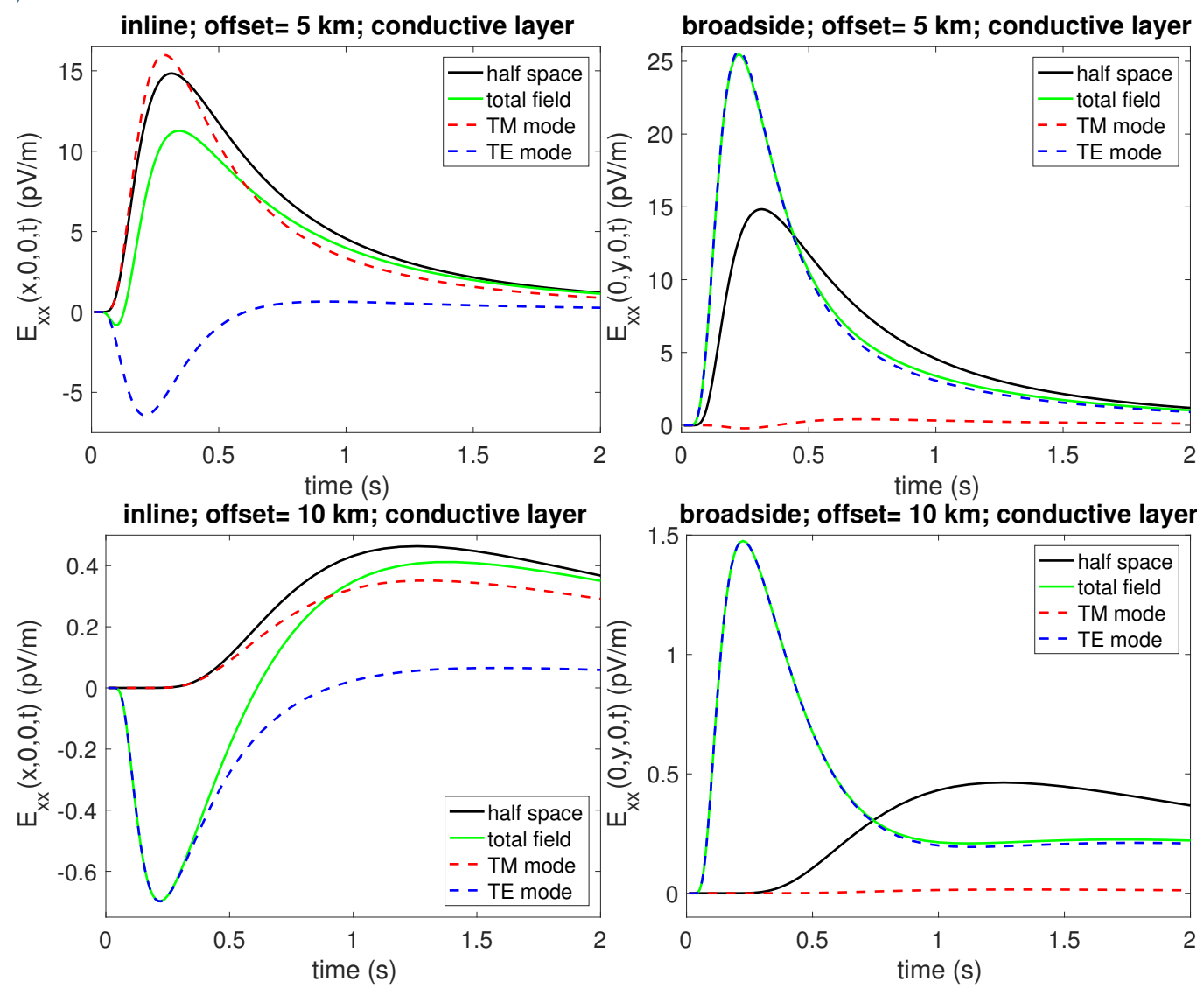

Figure 2 Inline (left) and broadside (right) electric field impulse responses at $5 \mathrm{~km}$ (top) and $10 \mathrm{~km}$ (bottom) offsets as a function of time for a buried conductive layer; the half space response is in black and the response with the layer is green, with TE mode in blue and TM mode in red.

by the TM mode whereas the broadside response is mostly determined by the TE mode. The presence of the resistive layer in the TM mode results primarily in increased amplitude but also in reduced arrival time of the peak amplitude at $10 \mathrm{~km}$ offset in the inline data. At $5 \mathrm{~km}$ offset the diffusion time along the vertical path down from the source, then horizontal through resistive layer and vertically up to receivers has an expected time of peak at $t=217 \mathrm{~ms}$ and we find it at $t=224 \mathrm{~ms}$ when we look at the peak time of the difference signal between half space and total field in the inline field This leads to an estimated layer resistivity of $137 \Omega \mathrm{m}$. At $10 \mathrm{~km}$ offset this is no longer the case, which suggests that there can be constructive interference inside the resistive layer of internal multiples that contribute to longer diffusion times. This would be helpful to resolve the thickness of the resistive layer in inversion. The information about the presence of the resistive layer is in broadside also the TM mode, because we can see in the bottom left graph that the TE mode response almost fully overlaps the half space response. At early times the TE mode at $10 \mathrm{~km}$ offset both in line and broadside gives a good indication of depth with an early peak at $\mathrm{t}=195 \mathrm{~ms}$ leading to an estimated depth of $1970 \mathrm{~m}$. Because it is visible in the total field, this is a helpful piece of information.

\section{Conclusions}

The results show that the presence of a resistive layer is detected best with the inline configuration and the information comes primarily from the TM mode part of the field, while the presence of a conductive layer is detected best with the broadside configuration and the information comes primarily form the TE mode part of the field. Simple geometrical considerations for diffusive propagation paths that lead to the electrically shortest paths from source to receiver allow for estimating medium properties with reasonable errors. We also found it likely that inside the resistive layer more complicated geometrical paths should exist that contribute to the measured response in the inline TM mode contribution. These can be helpful in inversion to estimate the layer thickness. 

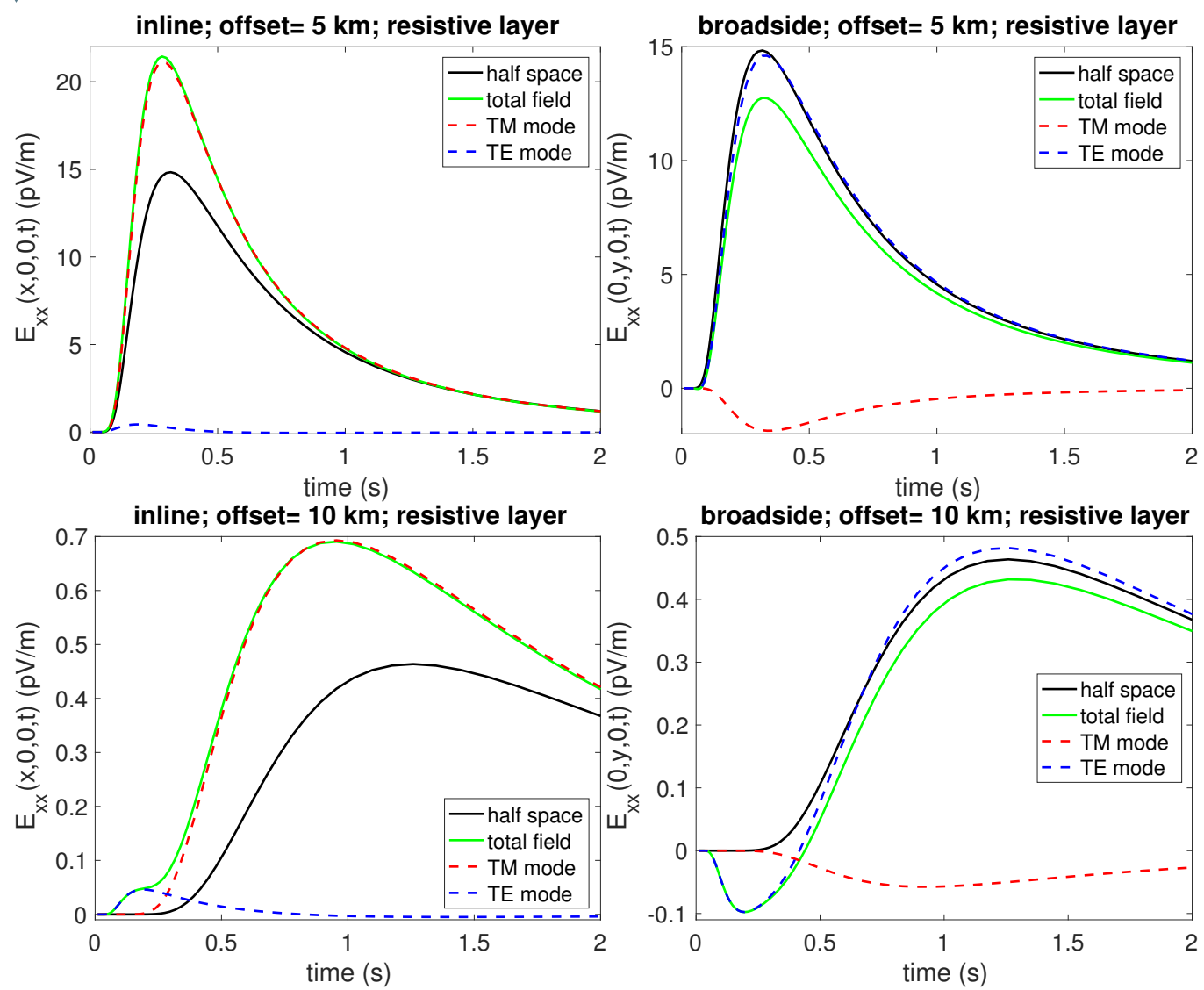

Figure 3 Inline (left) and broadside (right) electric field impulse responses at $5 \mathrm{~km}$ (top) and $10 \mathrm{~km}$ (bottom) offsets as a function of time for a buried resistive layer; the half space response is in black and the response with the layer is green, with TE mode in blue and TM mode in red.

\section{References}

den Boer, E., Eikelboom, J., van Driel, P. and Watts, D. [2000] Resistivity imaging of shallow salt with magnetotellurics as an aid to prestack depth migration. First Break, 18, 19-26.

Hanssen, P., Nguyen, A.K., Fogelin, L.T.T., Jensen, H.R., Skarø, M., Mittet, R., Rosenquist, M., Súilleabháin, L.O. and van der Sman, P. [2017] The next generation offshore CSEM acquisition system. In: SEG International Exposition and 87th Annual Meeting. SEG, Tulsa, 1194-1198.

de Hoop, A.T. [1996] A general correspondence principle for time-domain electromagnetic wave and diffusion fields. Geophysical Journal International, 127, 757-761.

Hunziker, J., Thorbecke, J. and Slob, E. [2015] The electromagnetic response in a layered vertical transverse isotropic medium: A new look at an old problem. Geophysics, 80(1), F1-F18.

Mittet, R. [2015] Seismic wave propagation concepts applied to the interpretation of marine controlledsource electromagnetics. Geophysics, 80(2), E63-E81.

Panzner, M., Morten, J.P., Weibull, W.W. and Arntsen, B. [2016] Integrated seismic and electromagnetic model building applied to improve subbasalt depth imaging in the Faroe-Shetland basin. Geophysics, 81(1), E57-E68.

Sheard, S.N., Ritchie, T.J., Christopherson, K.R. and Brand, E. [2005] Mining, environmental, petroleum, and engineering applications of electromagnetic techniques in geophysics. Surveys in Geophysics, 26, 653-669.

Slob, E., Hunziker, J. and Mulder, W.A. [2010] Green's tensors for the diffusive electric field in a VTI half-space. Progress in Electromagnetics Research-PIER, 107, 1-20.

Werthmüller, D. [2017] An open-source full 3D electromagnetic modeler for 1D VTI media in Python: empymod. Geophysics, 82(6), WB9-WB19.

Ziolkowski, A., Hobbs, B.A. and Wright, D. [2007] Multitransient electromagnetic demonstration survey in France. Geophysics, 72(4), F197-F209. 